\title{
EU Including: Development of Radiological and Nuclear Training Learning Objectives
}

\author{
Friedrich Steinhäusler \\ International Security Competence Centre GmbH, Baden, Austria \\ Email: steinhaeusler@isccentre.at
}

How to cite this paper: Steinhäusler, F. (2021) EU Including: Development of Radiological and Nuclear Training Learning Objectives. Journal of Applied Mathematics and Physics, 9, 2170-2178. https://doi.org/10.4236/jamp.2021.98136

Received: July 17, 2021

Accepted: August 28, 2021

Published: August 31, 2021

\begin{abstract}
Worldwide, about 20 million consignments of radioactive material are transported annually on public roads, railways, aircraft, and ships. About $95 \%$ of radioactive consignments are not related to nuclear power. In 2016, a total of 143 incidents of nuclear or other radioactive materials were found to be outside of regulatory control, which occurred in 19 countries. On an international level risk assessment has to account for the potential threats due to millions of radioactive sources in use worldwide and hundreds of tons of military grade $\mathrm{U} / \mathrm{Pu}$ not under IAEA safeguards. The European Union (EU) has tasked the INCLUDING project consortium, connecting 15 partners from 10 EU Member States, to address this issue and create an innovative cluster for radiological and nuclear $(\mathrm{RN})$ emergencies. The project is coordinated by the Italian Agency for the New Technologies, Energy and Sustainable Economic Development (ENEA). INCLUDING will provide comprehensive training in the RN security sector. Thereby, know-how is enhanced for practitioners in this sector. An important part in this endeavor is the development of radiological- and nuclear training learning objectives. INCLUDING partners involved in this task (Work Package 4) represent companies, organisations and government agencies from Austria, Greece, Italy, Lithuania, Hungary and Portugal. The task has four main objectives: 1) Harmonisation of RN education/training for EU first responders: 2) Identification of main problems in setting norms; 3) Developing a training matrix using revised Bloom's taxonomy; 4) Use of the methodology developed for Joint Actions and its application at INCLUDING Cluster Facilities in different EU Member States. The INCLUDING Work Package 4 members have analyzed the EU EDEN Training Matrix and identified gaps in accordance with NATO CBRN training standards related to civil-military cooperation. Furthermore, they analyzed 5 EUHORIZON 2020- and 9 EUFP7-SECURITY projects, and $97 \mathrm{RN}$ training courses offered to the international community by NATO, 6 EU organisations, Qatar, US military- and civilian organisations, and the International
\end{abstract}


Atomic Energy Agency (IAEA). This paper will present these results, which are being used to develop the basic structure for the Learning Objective Catalogue (LOC), comprised of multiple RN-related Learning Objectives for different threat scenarios.

\section{Keywords}

Radiological Emergency, Nuclear Emergency, Training, Learning Objectives

\section{Introduction}

Worldwide, about 20 million consignments of radioactive material are transported annually on public roads, railways, aircraft, and ships [1]. About $95 \%$ of radioactive consignments are not related to nuclear power. In 2016, open source reports found a total of 143 incidents of nuclear or other radioactive materials outside of regulatory control, which occurred in 19 countries; between 2013 and 2016 researchers have identified a total of 683 incidents occurring in 48 countries [2]. On an international level, risk assessment has to account for the potential threats due to millions of radioactive sources in use worldwide and hundreds of tons of military grade U/Pu not under IAEA safeguards. The European Union (EU) has tasked the consortium of the EU INCLUDING project ("Innovative Cluster for Radiological and Nuclear Emergencies"; https://including-cluster.eu/news.php), connecting 15 Partners from 10 EU Member States, to address this issue and create an innovative cluster for radiological and nuclear (RN) emergencies [3]. The project is coordinated by the Italian Agency for the New Technologies, Energy and Sustainable Economic Development (ENEA).

INCLUDING will provide comprehensive training in the RN security sector, enhancing practically applicable know-how for practitioners in the RN security sector. The main objectives of INCLUDING are:

- To provide practitioners in the RN security sector with an innovative European cluster pursuing a Pan-European Federated model to optimize sharing of resources and expertise, and paving the way for a certified collective membership by easing inter-facilities access to members of the Federation, and smooth the way for equipment circulation.

- To develop a centralized improved management tool for remote booking and utilization of resources in the Federation, joint engagement in training sessions development and post-event assessment. In the Federation individual Members will cooperate to provide a common framework to standardize access to their respective facilities, enhance interoperability and to allow a more intensive use of expensive equipment.

- To contribute to the development of a common learning framework for RN training and to facilitate the uptake and integration of new technologies and methodologies for practitioners in the RN field. Thereby, participants capi- 
talize on results and training facilities developed in previously funded EU and national projects.

- To execute Joint Actions (drills, multidisciplinary field exercise, table-top exercise, training sessions, instrument testing, etc.) to validate the Federated model and whose scenarios are developed, following the reconnaissance of emerging RN unconventional threats, and to bridge gaps in the specific exercise, simulation and training functions.

- To collaborate with other EU projects and Clusters and plan future developments enabling long-term cooperation and future Federation enlargement and sustainability.

An important part in this project is the development of $\mathrm{RN}$ training learning objectives.

The following European institutions are involved in this task assigned to INCLUDING Work Package 4: International Security Competence Centre (ISCC, Austria; Task Leader), Nuclear Security Centre of Excellence (NSCOE, Lithuania), Agencia Nazionale per le Nuove Tecnologie, L'energie e lo Sviluppo Economico (ENEA), Italy), Instituto de Engenharia de Sistemas e Computadores, Technologia e Ciencia (INESC TEC, Portugal), TEKEVER ASDS (TEKEVER, Portugal), Ministry of National Defence of Greece (HMOD, Greece), Policia de Seguranca Publica Portuguesa (PSP, Portugal), and Magyar Tudomanyos Akademia Energia Tudomany Kutaokozpont (MTA, Hungary).

The task has four main objectives: 1) Harmonisation of RN education/training for EU first responders; 2) Identification of main problems in setting norms; 3) Developing a training matrix using revised Bloom's taxonomy; 4) Use of the methodology developed for Joint Actions and its application at INCLUDING Cluster Facilities in different EU Member States. For this purpose, the consortium has analyzed the EU EDEN Training Matrix and identified gaps in accordance with NATO CBRN training standards related to civil-military cooperation.

The paper presents these results, which are used to develop the basic structure for the Learning Objective Catalogue (LOC). LOC is comprised of RN-related Training Learning Objectives for different threat scenarios. The framework created by the INCLUDING consortium will create constructive synergies among all the stakeholders, namely practitioners (police, fire brigade, customs, border guards, defence, regulators, radiation protection experts, operators/managers of training facilities, and innovation developers (academia, research centers, industries, Small/Medium Sized Enterprises (SMEs)). Thereby, resources will be optimized in Europe and associated countries.

\section{Methodology}

The project foresees three tasks to be fulfilled in Work Package 4, i.e., 1) Conduct of public workshops dedicated to the issue of RN threats, agencies and organisations involved, and challenges for first responders in getting the situation 
under control; 2) Development of training learning objectives, which can be applied to any radiological and nuclear threat scenario; 3) Validation and feedback from field tests (Joint Actions), conducted jointly by several dedicated organisations in different locations in the European Union.

\subsection{Workshops on the EU RN Training Framework}

Hitherto, the INCLUDING consortium held two workshops:

- 1st Workshop on Rad. \& Nucl. Training Framework in EU, Frascati (Italy), 8 Oct. 2020. It took place as a virtual meeting due to COVID 19 restrictions (organizer: Project Coordinator ENEA) and focused inter alia on: open issues in nuclear security; management of a radiological crime scene and problems in medical aspects posed by specific threat scenarios; recent events, where radiation environmental monitoring has been key in assessing the possible onset of a radiological emergency. Further details at https://including-cluster.eu/videos/video annualworkshop.mp4.

- 2nd Workshop on Rad. \& Nucl. Training Framework in EU, Athens (Greece), 24-25 June 2021 (organizer: National and Kapodistrian University of Athens). The Workshop was devoted to nuclear security issues, focusing on maritime infrastructure (primarily ports) and related open issues in prevention, detection and response to nuclear security scenarios. Further details at https://including-cluster.eu/news-post.php?id=31.

The results of these workshops covered strategic issues and the international legal framework, operational capabilities and innovation, as well as cooperation between EU projects and international activities.

\subsection{Development of RN Training Learning Objectives}

Learning objectives should be measurable to allow the delivery of a unified evaluation methodology within the wide range of training activities and scenarios. The method used in INCLUDING is inspired by the modular approach already adopted in the International CBRN Training Curriculum issued by NATO, and will further expand the training matrix concept introduced in EDEN; further details at (https://www.nato.int/docu/cep/cep-cbrn-training-e.pdf).

The training matrix allows decomposing the scenario of a training activity into a combination of eight different modules (Prevention, on site initial assessment and actions, on scene protection, on scene decontamination, on scene medical aid, on scene detection, Command and control, Recovery). Each module is further broken down into operative functions, for example module On-site initial assessment and actions is subdivided into General risks assessment, Diffusion mechanisms and models, Key information from the scene, Public alert, Command decisions, and Mass evacuation.

INCLUDING considers the application of Bloom's taxonomy as important, since it ensures that lesson objectives are developing critical thinking and higher order cognitive abilities in students [4]. This approach differentiates between 
Knowledge based domain (KD), Emotion based domain (ED), and Action based domain (AD):

- KD: Recall or recognition of specific facts, procedural patterns, concepts that serve in the development of intellectual abilities and skills, e.g., understand, evaluate,

- ED: Describes the ways people react emotionally and their ability to feel other living pain or joy, e.g., respond to phenomena, valuing,

- $\mathrm{AD}$ : Describe the ability to physically manipulate a tool or instrument, e.g., origination, guided response.

As an example, a possible scheme to develop learning objectives for the operative function Triage, module On-scene medical aid, is applied in the following manner:

Domain: Knowledge, Action, Emotion;

Level: Evaluate, create, complex response, receiving phenomenon, apply;

Learning Objectives: Check early clinical symptoms of exposure to radiation; generate a report on symptoms of the patient; measure key physiological parameters (blood pressure, heart rate, temperature, ECG, etc.); assist the patient psychologically; implement outpatient surveillance or hospitalization in a department of hematology or radiopathology.

\subsection{Validation and Feedback from Joint Actions}

INCLUDING is creating an international network of specialized facilities in the Member States of the European Union. At these facilities, Joint Actions (JA) are implemented, encompassing field exercises, resident or remote training sessions, and testing of equipment. All activities are organized in the INCLUDING network of facilities and by exploiting their existing resources. The INCLUDING Platform is the operative tool to optimize the execution of the JA through expertise and resource sharing. The Project Coordinator and a consortium partner constitute the Joint Action Board (JAB), refining the scenario and objectives. By uploading it on the INCLUDING Platform (web-based) it is made accessible to all partners. The JAP addresses all aspects of the action, including:

Plan for preparatory activities; Scenario and objectives; Description of the activities and technical solutions; Safety rules; Logistic information; Ethical issues; Evaluation methodology and evaluation criteria.

Hitherto, the INCLUDING consortium has prepared in detail the following JAs:

1) JA Athens (Greece), 22-23 June 2021:

The Athens Joint Action was a multidisciplinary field exercise, dealing with counteracting an attempt to smuggle radioactive sources from a port (Piraeus commercial port). It was assumed that an unidentified container contains an unknown radiation source (Caesium-137). During the exercise Unmanned Aerial Vehicle (UAV)/Unmanned Ground Vehicle (UGV) technology has been deployed. Furthermore, the interoperability of the tools shared in the INCLUDING 
Federation has been checked. It was a full-scale exercise, designed to establish a learning environment for participants to be trained in emergency response plans, policies and procedures in such situations. The exercise was conducted in a no-fault learning environment, i.e., systems and processes, not individuals, have been evaluated. Exercise simulation has been realistic and plausible, containing sufficient detail from which to respond. Exercise participants have reacted to the information and situations as they were presented, in the same manner as if this had been a real event. The main aim of the exercise was:

- Conduct initial assessment (size-up) with emergency response services arriving promptly at the scene. Establishment of a safe perimeter confirmed as safe by scene survey and measurements of contamination.

- Deployment of UAVs/UGVs for detection/identification purposes. Accurate and timely dissemination of protective action messages to emergency personnel.

- Implementation of the Incident Command System (ICS) and on-scene management. Seamless and effective transfer of responsibility at the site.

- Maintaining personnel accountability system. Measures to prevent the spread of contamination.

- Coordination and support of decontamination activities on-site. Survey of the incident scene. Capability of continuous monitoring, dose evaluation, and radiation protection. Ability to provide adequate medical care for personnel affected by the emergency conditions.

- Prepare post-emergency reports. Reliability of and the effective use of emergency communications equipment, communication procedures and methods. Establishment of perimeter, Hot, Warm and Cold Zones. Decision making based on realistic data and real weather conditions.

- Addressing the issue of hybrid threats.

- Producing public information releases.

2) JAMikkeli (Finland),13-16 September 2021:

The JA FTX Mikkeli (Demo Live, Table-top exercise, Virtual Reality/serious gaming, hosted by South-Savo Fire Department (SSAV)) is a multi-agency field technical exercise against an $\mathrm{R} / \mathrm{N}$ incident scenario caused by stolen and missing radioactive orphan source (Caesium-137). It is assumed that civilians are exposed accidentally to the radiation source. Several local authorities are involved in the hazardous situation management. The exercise is developed to test a regional and country's current capabilities and identify areas for improvement in case of an $\mathrm{RN}$-incident. The exercise tests coordination mechanisms, identifies requirements for scientific support, and validates practices to share information. The main aims tested in the field are:

- Concepts and operations in a radiological orphan source incident.

- Elements on how to establish a command structure and command chain for a radiological orphan source incident, and apply IAEA guidelines, including crime scene management. 
- Elements of practical use of radiation detectors and UAVs for operational purposes. Elements of establishing decontamination lines, First Aid Station and procedures.

- Elements on management of information flow in a nuclear security event.

3) JA Bologna (Italy), scheduled for Spring 2022:

The threat scenario in the Bologna Joint Action foresees the intentional irradiation of a large number of citizens in an urban environment. A fit-for-purpose Table-Top Exercise (TTX) deals with the management and response to a terror attack in the densely inhabited urban area of Bologna. The scenario in the TTX is concerned with an emergency on the Italian territory that evolves at provincial/regional scale without putting at risk national interests and that does not overwhelm the national response capabilities. It is based on the Italian framework for responding at the strategic, tactical and operative level to an $\mathrm{RN}$ emergency. The main aims of the TTX will be:

- To present to the participants all the elements of the whole chain of decisions typically taken during an emergency. This includes the constraints due to the urgency of taking decisions, since the exercise will be almost in real-time (stress factor).

- To introduce novel technologies and to demonstrate their usefulness in dealing with such scenarios, such as: numerical codes to predict atmospheric dispersion of pollutants in dense urban areas; quickly deployable unmanned and automated set of devices (sensors) to measure the contamination on the field; devices for screening tests of externally or internally contaminated persons; a network of automated spectrometric detectors deployed on public transit.

- To share and exchange views, ideas and opinions during the TTX itself on how certain decisions are taken in different countries, discussing the different approaches and practices, especially on how to deal with some issues and specificities of radiological dispersal devices used in densely inhabited urban areas.

\section{Learning Objective Catalogue (LOC)}

A key component in the INCLUDING project is training and associated learning. In this regard, consortium members analyzed hitherto 5 EU HORIZON 2020- and 9 EU FP7-SECURITY projects, and $97 \mathrm{RN}$ training courses offered to the international community by NATO, 6 EU organisations, Qatar, US military and civilian organisations, and the International Atomic Energy Agency (IAEA). Currently, more than 250 learning objectives have been identified. These objectives can be categorized as follows:

- Biological and medical effects of radiation. Goal: Principles of management of RN casualties.

- Radiation Detection. Goal: Identification of radioactive material.

- Radiation Protection. Goal: Installation of radiation safety programme management. 
- Survey. Goal: Planning and preparing for reconnaissance operations.

- Decontamination. Goal: Principles of personal protection, agent detection, recognition and emergency treatment of agent exposure.

- Radiological and Nuclear Terrorism. Goal: Identification of the difference between RN incidents and other hazardous material incidents, and associated countermeasures.

- Nuclear Emergencies. Goal: Familiarization of participants with the various elements of nuclear emergencies.

- Incident Commander. Goal: Understand the basics of the incident commanders' tasks and responsibilities.

- Emergency Preparedness Coordinator. Goal: Assist organisations in managing exercises by testing preparedness for nuclear or radiological emergencies.

- Response Processes and Capabilities. Goal: Planning and response considerations for reconnaissance operations in an $\mathrm{RN}$ event.

Each of these categories contains several specific learning objectives. In order to provide a structure for future practical applications of the learning objectives, the Learning Objective Catalogue (LOC) is being established. The LOC offers a tailor-made set of learning objectives for different $\mathrm{RN}$ threat scenarios, e.g., deployment of a radiological dispersal device (any device that causes the purposeful dissemination of radioactive material without a nuclear detonation), radiation exposure device (intentional irradiation of individuals), weapon of mass disturbance (intentional contamination of bank notes, clothing, food, etc.), nuclear accident (nuclear power plant), and weapon of mass destruction (detonation of a crude nuclear device).

\section{Conclusions}

Managing the aftermath of $\mathrm{RN}$ threats require different skills from a wide spectrum of experts and dedicated organizations. $\mathrm{RN}$ training courses for these groups need to address appropriate learning objectives of response methods (search, response, consequence management) to a variety of nuclear and radiological incidents. This covers a wide range from a small, localized radioactive material release to a larger incident, such as a major accident (International Nuclear Event Scale exceeding level 5) at a nuclear facility.

The INCLUDING approach analyzes the learning objectives offered by different countries and organizations, and creates a structured overview in the LOC for future applications in different training courses. The results assist in achieving interoperability among first responders in an international response to an RN event. Standardized training of RN staff personnel, in compliance with the LOC, ensures that trainees can address biological and medical effects of radiation, radiation detection and -protection, survey methods, decontamination techniques, threats due to radiological and nuclear terrorism, nuclear emergencies, understand the role of the incident commander, of the emergency preparedness coordinator, and comprehend the multifaceted response processes and 
capabilities.

\section{Acknowledgements}

The research leading to these results has received funding from the European Union's Horizon 2020 Research and Innovation Programme under Grant Agreement No. 833573 (https://including-cluster.eu).

\section{Conflicts of Interest}

The author declares no conflicts of interest regarding the publication of this paper.

\section{References}

[1] World Nuclear Association, Transport of Radioactive Materials. https://www.world-nuclear.org/information-library/nuclear-fuel-cycle/transport-of -nuclear-materials/transport-of-radioactive-materials.aspx

[2] James Martin Center for Nonproliferation Studies (CNS), Global Incidents and Trafficking Database, 2016 Annual Report.

https://media.nti.org/documents/global incidents and trafficking 2016.pdf

[3] INCLUDING Project, Innovative Cluster for Radiological and Nuclear. https://including-cluster.eu/

[4] Hamada, S. (2021) What Is Bloom's Taxonomy? http://www.bloomstaxonomy.us/ 